\title{
Application of Comprehensive Nursing Mode in Patients with Acute Ischemic Stroke After Intra-arterial Intervention
}

\author{
Peng Zhang ${ }^{1}$, Min Xie ${ }^{2}$, Xiao Yong Dai ${ }^{1, ~ * ~}$ \\ ${ }^{1}$ Interventional Operating Room, The First Affiliated Hospital of Jinan University, Guangzhou, China \\ ${ }^{2}$ Neurology Department, The First Affiliated Hospital of Jinan University, Guangzhou, China \\ Email address: \\ zhangp9863@qq.com (Peng Zhang),1721444577@qq.com (Min Xie), 1045526110@qq.com (Xiao Yong Dai) \\ ${ }^{*}$ Corresponding author
}

To cite this article:

Peng Zhang, Min Xie, Xiao Yong Dai. Application of Comprehensive Nursing Mode in Patients with Acute Ischemic Stroke After Intra-arterial Intervention. American Journal of Nursing Science. Vol. 10, No. 1, 2021, pp. 24-27. doi: 10.11648/j.ajns.20211001.15

Received: December 24, 2020; Accepted: January 11, 2021; Published: January 18, 2021

\begin{abstract}
Objective: To explore the application of comprehensive nursing mode in patients with acute ischemic stroke after intra-arterial intervention. Methods: 218 patients with acute ischemic stroke undergoing interventional surgery from January 2019 to December 2019 were randomly divided into a control group (routine nursing mode, $\mathrm{n}=109$ ) and a study group (comprehensive nursing mode, $\mathrm{n}=109$ ). The control group adopts conventional nursing mode, such as basic nursing, life nursing, safety nursing, etc. The research group adopts the comprehensive nursing mode. Before the implementation of nursing measures, the patients' vital signs, state of consciousness, operation process and mental state are comprehensively evaluated to understand the actual needs of the patients. In the course of patient care, through real-time observation and evaluation, in a multidisciplinary cooperative mode to implement integrated care. According to the National Institutes of Health Stroke Scale (NIHSS), the two groups of patients were scored respectively after intervention and 1 week after intervention in order to compare the neurological function of them. Results: The NIHSS score of the study group 1 week after intervention was lower than that of the control group, and the difference was statistically significant $(\mathrm{P}<0.05)$. Patients in the study group achieved better clinical rehabilitation effect than those in the control group. Conclusion: The comprehensive nursing mode can improve the prognosis of patients, which is worthy of promotion in clinical practice.
\end{abstract}

Keywords: Acute Ischemic Stroke, Interventional Therapy, Comprehensive Nursing Mode

\section{Introduction}

Cerebral stroke is a group of diseases characterized by cerebral ischemia and hemorrhagic injury. It is also called "stroke" or "Cerebrovascular accident". It has three notable characteristics: high incidence rate, high disability rate and high mortality rate. It is the first cause of death and disability in adults in China [1]. Among them, Acute Ischemic Stroke (AIS) accounts for $80 \%$ of the total. It is important to open the occluded vessels and save the ischemic penumbra as soon as possible. The earlier the intervention, the better the recovery of brain injury and the less neurological sequelae. The 2015 edition of the Chinese Guidelines for Endovascular Treatment of Acute Ischemic Stroke has listed "endovascular embolectomy" as grade I recommendation for AIS [2]. which brings new direction and hope to the treatment of this disease. But at the same time, it also brings new requirements and challenges to nursing work. In this study, the comprehensive nursing mode was applied to the nursing care of patients with acute ischemic stroke. The detailed report is as follows.

\section{Data and Methods}

\subsection{Clinical Data}

218 patients with acute ischemic stroke undergoing interventional surgery from January 2019 to December 2019 were selected as the research objects. All patients were diagnosed as acute ischemic stroke by CT and DSA, and endovascular embolectomy was performed in all patients. All cases were divided by random number table method into a control group and a study group with 109 cases in each group. 
There were 73 male patients and 36 female patients in the control group, aged from 27 to 89 years old $(60.84 \pm 12.95)$; in the study group, there were 70 male patients and 39 female patients, aged from 40 to 85 years old $(64.96 \pm 13.28)$. There was no statistical significance in general data between the two groups $(\mathrm{P}>0.05)$.

\subsection{Nursing Methods}

\subsubsection{The Control Group}

Adopting routine nursing mode.

\subsubsection{The Study Group}

Adopting the comprehensive nursing mode with patients as the center in combination of departments such as neurosurgery department, interventional vascular department, rehabilitation department, psychological department.

i. comprehensive evaluation of patients before implementation of nursing measures

In addition to the general data, vital signs, consciousness, swallowing function, activities of daily living, psychological and mental state of patients, matters related to interventional surgery, such as lateral appendage puncture, prevention of complications were all evaluated before the implementation of nursing measures.

ii. monitoring and maintenance of vital signs of patients

The vital signs of patients after intervention need to be comprehensively observed and recorded, including their consciousness, pupil, electrocardiogram, blood pressure, oxygen saturation, muscle strength, blood glucose, etc. Patients with deep disturbance of consciousness, irritability, vomiting and other symptoms, suggesting intracranial hemorrhage or high perfusion syndrome. It should be reported to the doctor immediately for further treatment according to the patient's head CT. [3] According to the guidelines, blood pressure should be controlled within $180 / 105 \mathrm{mmHg}$ during mechanical thrombectomy and within 24 hours after the end of treatment (grade IIa recommendation, grade B evidence); systolic blood pressure should be controlled below $140 \mathrm{mmHg}$ after vascular recovery and reperfusion (grade $\mathrm{IIb}$ recommendation, grade B evidence). [4]

iii. nursing of punctured lateral appendage

After the implementation of intra-arterial interventional therapy, the patient's femoral artery puncture point needs to be strictly bandaged and immobilized. At present, elastic bandage is usually used for cross fixation and immobilization of puncture points for $6 \mathrm{~h}$, and patients who can't cooperate will be restrained if necessary. For patients with indwelling arterial sheath, blood was drawn back regularly to observe whether there was thrombosis. At the same time, the sheath tube was flushed with $50 \mathrm{unit} / \mathrm{ml}$ heparin saline q6h to prevent the formation of thrombosis. Closely observe the skin and surgical dressing around the puncture point to see whether there is bleeding, ecchymosis and hematoma. Due to the pressure bandaging on the puncture point, it is necessary to strengthen the observation of skin temperature, skin color and dorsalis pedis artery of patients during nursing [5], so as to prevent lower limb artery ischemia. As the patient was retained in bed, there was a potential risk of deep venous thrombosis of lower extremity. [6]It is necessary to guide and assist the patient with ankle pump exercise to promote venous blood circulation of lower limbs.

iv. medication care

After the operation, patients should take the medicine on time and in accordance with the advised amount under medical supervision. As the patients who use antiplatelet and anticoagulant drugs after AIS intra-arterial interventional therapy have a greater risk of bleeding, so it is necessary to observe whether there are symptoms such as gingival bleeding, black stool and urethral bleeding.

v. care for diet and swallowing function

Dysphagia is one of the common complications of cerebrovascular disease. According to domestic and foreign literature studies, the incidence of dysphagia after stroke is as high as $51 \% \sim 73 \%$; $1 / 3$ of dysphagia patients will have aspiration, and half of the patients have recessive aspiration without cough [7]. The resulting aspiration pneumonia and asphyxia seriously reduce the quality of life of patients, and even threaten their life safety. In nursing work, assessment on dysphagia is particularly important. There are two main clinical evaluation methods: Repetitive Saliva Swallowing Test (RSST) and Water Swallow Test. For patients with dysphagia, we should strengthen diet education, guide them to adopt appropriate body position (generally, at least $30^{\circ}$ supine position of trunk and forward head flexion, food and swallowing skills. At the same time, rehabilitation department needs to join in to guide patients to carry out swallowing rehabilitation exercise as soon as possible. There is a study has pointed out that the comprehensive nursing mode for patients with dysphagia after stroke has a positive effect on restoring the function of swallowing and improving the nutritional status. [8]

vi. observation and nursing of complications of intra-arterial interventional therapy

Due to the specificity of intra-arterial procedures, patients undergoing endovascular intervention may have related complications, among which hemorrhagic transformation is one of the main complications of AIS thrombolysis or endovascular treatment. In the recent positive results test, the incidence of Spontaneous Intracranial Hemorrhage (SICH) was $1.9 \%-7 \%$. [4] Other complications, such as hyperperfusion syndrome, contrast allergy, contrast induced nephropathy would affect the prognosis of patients. Some studies have proved that evidence-based nursing can effectively reduce the incidence of complications and improve their life quality after treatment. [9]Therefore, it is particularly important to observe the complications of patients undergoing intra-arterial interventional therapy. Nursing staff should identify the complications early and take evidence-based nursing measures with critical thinking.

vii. psychological nursing and support of patients

Post-stroke depression (PSD) is one of the most common emotional disorder syndromes. [10] PSD can prolong the length of hospital stay, aggravate the degree of neurological impairment, and even increase the mortality rate. PSD 
patients have a 3.4 times greater risk of dying within 10 years than non-depressed patients. [11] Some studies have shown that psychological nursing for patients with acute ischemic stroke can effectively improve their psychological state and ensure better prognosis of treatment. [12] In the psychological nursing of patients, it is necessary to strengthen the communication with patients, obtain their trust, establish a good relationship with them, enhance their confidence to overcome the disease, and timely observe their emotional changes and causes so as to apply targeted interventions. It is also necessary to identify the patients who may have psychological disorders, At present, there is no unified clinical psychometric index for post-stroke depression. [13] In the study group, we use the mental health scale to score the degree of depression, and if necessary, work with the psychiatry department for intervention.

\subsection{Evaluation Index}

According to the National Institutes of Health Stroke Scale (NIHSS), two groups of patients were scored after intervention and 1 week after intervention.

\subsection{Statistical Methods}

SPSS22.0 Statistical Software was used for statistical processing. Repeated measurement ANOVA and t test were used for measurement of normal distribution data, and rank-sum test was used for measurement of non-normal distribution data. Chi-square test was used for counting data. $\mathrm{P}<0.05$ means the difference is statistically significant.

\section{Results}

There was no statistical significance in the score difference between the two groups $(\mathrm{P}>0.05)$; the NIHSS score of the study group was lower than that of the control group one week later, and the difference was statistically significant $(\mathrm{P}<0.05)$, as shown in Table 1 .

According to the comparison of the two groups of data, the patients who adopted the comprehensive nursing mode achieved better clinical rehabilitation effect.

Table 1. Comparison of NIHSS scores of two groups one week later after intervention.

\begin{tabular}{llll}
\hline Groups & Cases & After intervention & One week after intervention \\
\hline Control group & 109 & $15.56 \pm 4.26$ & $7.69 \pm 2.45$ \\
Study group & 109 & $16.20 \pm 4.33$ & $9.53 \pm 2.16$ \\
P value & & 0.068 & 0.037 \\
\hline
\end{tabular}

Note: NIHSS, National Institutes of Health Stroke Scale

\section{Discussion}

Endovascular intervention applied to patients with acute ischemic stroke can quickly open the blood vessels. After operation, nursing staff should have rich professional knowledge and critical thinking. The core of the patient-centered multidisciplinary integrated nursing mode is to establish a decision-making platform with neurology as the core to provide continuous, standardized and professional nursing for patients in the whole process. [14]

The establishment of comprehensive nursing mode should be based on evidence-based nursing. [15] According to the principle of effectiveness, targely put forward effective nursing measures for patients with acute ischemic stroke to help patients get a better clinical prognosis.

\section{Conclusion}

The implementation of comprehensive nursing mode for patients with acute ischemic stroke after operation is also very consistent with early rehabilitation concept proposed now. This provides a way of comprehensive nursing of stroke patients, and has positive significance for improving the prognosis. Therefore, it is worth popularizing and applying in clinical practice.

\section{Acknowledgements}

In writing this article, I would like to thank my colleagues for their help, especially Professor Zheng Dongxiang's team, who helped me to collect the materials I needed and provided me with very important suggestions. Without their selfless dedication, I could not have finished this article.

I would also like to thank Professor Chen Lifang for her guidance. During the process of writing this article, she has been guiding me and providing me with good ideas. Besides, she has carefully read the manuscript and provided me with good suggestions for revision. I benefited a lot from her academic excellence.

My wife has given me a lot of care in my life as I write this, and she has also given me a lot of encouragement. And my two lovely sons, whose company makes my life more meaningful.

I also want to thank the Jinan University Library and its staff, without whose help none of this would have been possible.

\section{References}

[1] Wang Longde, Liu Jianmin, Yang Yi, et al. Great Challenges in Prevention and Treatment of Stroke in China-Summary of Chinese Stroke Prevention and Treatment Report 2018 [J]. Chinese Circulation Journal, 2019, 34 (2): 105-119.

[2] Gao Feng, Xu Anding. Chinese Guidelines for Endovascular Treatment of Acute Ischemic Stroke 2015 [J]. Chinese Journal of Stroke, 2015, 10 (7): 590.

[3] Zhou Lili, Lan Yan. Nursing Care of Patients with Acute Ischemic Stroke after Mechanical Thrombectomy [J]. Journal of Frontiers of Medicine, 2019, 9 (14): 174-175. 
[4] HuoXiaochuan, Gao Feng. Chinese Guidelines for Endovascular Treatment of Acute Ischemic Stroke 2018 [J]. Chinese Journal of Stroke, 2018, 13 (07): 706-729.

[5] Ding Qing, Zhou Shanshan, Jinping. Perioperative Nursing Experience of Mechanical Thrombectomy with Solitaire AB Stent in the Treatment of Acute Cerebral Infarction [J]. Anhui Medical Journal, 2018, 39 (2): 226-228.

[6] Zeng Z, Hu Z, Zhang J. Venous thromboembolism prevention during the acute phase of intracerebral hemorrhage [J]. Journal of the Neurological Sciences, 2015, 358 (1-2): 3-8.

[7] Hart TR, Paik NJ, Park JW. Quantifying swallowing function after stroke: A functional dysphagia scale based on videofluoroscopic studies [J]. Arch Phys Med Rehabil, 2001, 82 (5): 677-682.

[8] Luo Yanzhen, Luo Weihua, Lin Minying, Huang Huixian, Zhang Qizhi. Clinical Effect of Comprehensive Nursing Model on Swallowing Function and Nutritional Status of Patients with Dysphagia After Stroke [J]. China \&Foreign Medical Treatment, 2020, 39 (19): 123-125.

[9] Wang Shujun. Effect of Evidence-based Nursing on Complications Incidence and Life Quality of Patients with Cerebrovascular Disease Undergoing Interventional Therapy $[\mathrm{J}]$. International Journal of Nursing, 2020 (06): 1114-1115-1116-1117.
[10] Yu Sisi, Li Yun, Cai Minke. Study on the Effect of Short-term Solution focused Therapy on the Emotion of Patients with Post-stroke Depression [J]. Chinese Remedies and Clinics, 2020, 20 (6): 997-999.

[11] Niloufar H, Diane JT, Ruth L, et al. Post stroke depression and functional outcome: a critical review of litemture [J]. Heart Lung, 2009, 38 (2): 151-162.

[12] Yang Wenyu. Application Analysis of Psychological Nursing in Nursing Care of Patients with Acute Ischemic Stroke [J]. Medical Diet and Health, 2018 (4): 107-107.

[13] Jyotirekha D, Rajanikant GK. Post stroke depression: the sequelae of cerebral stroke [J]. Neurosci Biobehav Rev, 2018, 90 (12): 104-114.

[14] Li Chune, Zhang Xiaomei, Zhou Chongbin, Wan Qing, Zhang Xiaolin, Tang Xiaoxia. Effect of Multidisciplinary Team Cooperation Mode on Dysphagia in Patients with Acute Ischemic Stroke [J]. Yunnan Journal of Traditional Chinese Medicine and Materia Medica, 2020, 41 (04): 92-94.

[15] Liu Guiqin, Liu Hongjun, Li Jinxia, Li Lingyan, Wang Hao, Liu Jiankun.. Application of Comprehensive Nursing Intervention Based on Evidence in Middle-aged Patients with Ischemic Stroke [J]. Modern Journal of Integrated Traditional Chinese and Western Medicine, 2020, 29 (30): 3413-3416. 